\title{
Inoculación de Trichoderma longibrachiatum en algodón transgénico: Cambios en compuestos fenólicos y enzimas de estrés oxidativo
}

\author{
Inoculation of Trichoderma longibrachiatum in transgenic cotton: \\ change in the phenolics compounds and enzymes of oxidative stress \\ Tania Elisa González-Soto1, Lizbeth Moreno-Ramírez', Rosalba Troncoso-Rojas ${ }^{2}$, \\ Daniel González-Mendoza*1, Alberto Sánchez-Estrada ${ }^{2}$, Onecimo Grimaldo Juárez ${ }^{1}$, \\ Olivia Tzinzun-Camacho', Carlos Ceceña-Durán ${ }^{1}$
}

\section{RESUMEN}

El objetivo del presente estudio fue evaluar la actividad de las enzimas fenilalanina amonio-liasa (PAL), peroxidasa (POX) y la producción de compuestos fenólicos y flavonoides en plantas de algodón transgénico en respuesta a la inoculación con Trichoderma longibrachiatum (ICA-4). Noventa días después de la inoculación con T. longibrachiatum, hojas de las plantas de algodón fueron colectadas y las enzimas PAL, POX y los compuestos fenólicos fueron determinados. Los resultados mostraron que la inoculación aumentó el contenido de compuestos fenólicos y flavonoides en $18 \%$ y $45 \%$, respectivamente; comparadas con plantas no inoculadas. La actividad de las enzimas peroxidasa (4,88 Ux 10-5/mg de proteína), y fenilalanina amonia-liasa ( $0,0176 \mathrm{U} / \mathrm{mg}$ de proteína) mostraron diferencias significativas en comparación con la actividad enzimática de plantas no inoculadas $\left(3,48 \times 10^{-5} \mathrm{U} / \mathrm{mg}\right.$ proteína para POX y 0,01 U/mg de proteína para PAL). Estos resultados sugieren la inducción de una respuesta de resistencia sistémica inducida por la inoculación de las semillas con la cepa Trichoderma ICA-4.

Palabras clave: ruta fenilpropanoide, biocontrol, Trichoderma, enzimas de estrés oxidativo.

\section{ABSTRACT}

The objective of the present study was to evaluate the activity of phenylalanine ammonia-lyase (PAL), peroxidase (POX) and the production of phenolic compounds and flavonoids in transgenic cotton plants in response to inoculation with Trichoderma longibrachiatum (ICA-4). Ninety days after inoculation with $\mathrm{T}$. longibrachiatum, leaves of the cotton plants were collected and the enzymes PAL, POX and phenolic compounds were determined. The results showed that inoculation increased the content of phenolic compounds and flavonoids by $18 \%$ and $45 \%$, respectively, compared to non-inoculated plants. The activity of the enzymes peroxidase (4.88 Ux10-5/mg protein), and phenylalanine ammonia-lyase (0.0176 U/mg protein) showed significant differences compared to the enzymatic activity of uninoculated plants (3, 48x10-5 U/mg protein for POX and $0.01 \mathrm{U} / \mathrm{mg}$ protein for PAL). These results suggest the induction of a systemic resistance response induced by seed inoculation with the Trichoderma ICA-4 strain.

Key words: route phenylpropanoid, biocontrol, Trichoderma, enzymes of oxidative stress.

\section{Introducción}

El algodón (Gossypium hirsutum L.) es el cultivo de mayor importancia económica utilizado en la industria textil y constituye más de la mitad del consumo de fibra textil en todo el mundo (Karademir et al., 2011). El algodón
Bollgard (BG) contiene la toxina Cry 1Ac, la que actúa reduciendo la incidencia de gusano rosado (Pectinophora gossypiella) y el gusano bellotero (Heliothis Virescens), por lo que la siembra de este cultivar de algodón ha aumentado en el valle de Mexicali en los últimos años reduciendo la aplicación de insecticidas y proporcionado

1 Instituto de Ciencias Agrícolas de la Universidad Autónoma de Baja California (ICA-UABC). Ejido Nuevo León, Baja California, México.

2 Centro de Investigación en Alimentación y Desarrollo, A.C., Dirección de Tecnología de Alimentos de Origen Vegetal. Hermosillo, Sonora, México.

* Autor por correspondencia: danielg@uabc.edu.mx

Fecha de Recepción: 25 Septiembre, 2016.

Fecha de Aceptación: 2 Febrero, 2017.

DOI: $10.4067 / \mathrm{S} 0718-34292017005000005$ 
mayores rendimientos (Terán-Vargas et al., 2005; Vargas-Bejarano et al., 2012). Sin embargo, en el valle de Mexicali, el crecimiento de algodón puede ser afectado severamente por varios agentes de estrés bióticos (por ejemplo, hongos, virus e insectos). Dentro de estos factores la presencia de la marchitez del tallo, causada por hongos, es una enfermedad generalizada en el cultivo que se produce durante la etapa inicial de crecimiento cuando prevalecen condiciones de alta humedad (Rajendran y Samiyappan, 2008). Estudios recientes muestran que las plantas de algodón transgénicas que contienen la toxina Cry 1Ac, han mostrado ser más susceptibles a las enfermedades fúngicas en comparación con las líneas convencionales ( $\mathrm{Li}$ et al., 2009). Esto, debido a que los exudados de las raíces de algodón transgénico actúan estimulando la germinación de esporas y el crecimiento micelial de Fusarium oxysporum (Li et al., 2013). En este sentido, las estrategias para el manejo del marchitamiento por Fusarium incluyen el uso de cultivares y prácticas culturales. Sin embargo, la capacidad de sobrevivir de $F$. oxysporum en el suelo durante años o décadas hacen difícil controlar este microorganismo con el uso de cultivares o prácticas culturales (Zahoor et al., 2012).

Por lo tanto, es necesaria la búsqueda de alternativas biotecnológicas que permitan ayudar a controlar la marchitez causada por Fusarium. En este contexto, la expresión de resistencia sistémica inducida en plantas por la acción de microorganismos benéficos como bacterias y hongos, podrían ser una alternativa factible en el control de enfermedades vasculares en algodón. Estudios recientes reportan la eficacia de algunas especies del género Trichoderma como inductores de resistencia sistémica contra fitopatógenos (Chowdappa et al., 2013). Trichoderma spp., es un hongo antagonista con acción contra varios patógenos del suelo, tales como Rhizoctonia, Sclerotium, Fusarium y Pythium. Se asocia principalmente con la producción de las proteínas relacionadas con la patogénesis, como $\beta-1,3-$ glucanasa, compuestos fenilpropanoides y otros mecanismos relacionados con la defensa que se observan en plantas previamente colonizadas por diferentes cepas de Trichoderma (Harman et al., 2004; Nawrocka y Malolepsza, 2013). No obstante, el efecto de T. longibrachiatum en la producción de compuestos fenilpropanoides y enzimas oxidativas en plántulas de algodón transgénico ha sido poco estudiado. De esta forma, el objetivo de este estudio fue evaluar el efecto de la aplicación de Trichoderma longibrachiatum cepa ICA-4 en el contenido de compuestos fenólicos (fenoles y flavonoides totales), así como en la expresión de las enzimas fenilalanina amonio-defensa liasa y peroxidasa (enzimas oxidativas) en plantas de algodón transgénicas.

\section{Materiales y Métodos}

Los estudios se hicieron en el sitio experimental del Instituto de Ciencias Agrícolas de la Universidad Autónoma de Baja California, Baja California, México (32²8'5.63" N, 115'12'11.94" O), en el período de marzo a septiembre de 2014.

\section{Preparación del inóculo de T. longibrachiatum}

El inoculante se preparó con la inoculación de cinco mililitros de $T$. longibrachiatum ICA-4 cepa (GenBank: KJ190162) a una concentración de $1 \times 10^{8}$ esporas $\mathrm{ml}^{-1}$ en bolsas del arroz estéril $(150 \mathrm{~g})$ incubando por 15 días, período después del que se secó en un horno a $40{ }^{\circ} \mathrm{C}$, durante $24 \mathrm{~h}$. Las esporas se extrajeron y se mezclaron con talco (malla 350 con $95 \%$ de blancura) y $1 \%$ de sacarosa, hasta obtener una concentración de $1 \times 10^{6}$ esporas $\mathrm{g}^{-1}$.

\section{Inoculación de semillas de algodón y experimento de campo}

Usando una suspensión del inoculante a una concentración de $1 \times 10^{6}$ esporas $\mathrm{g}^{-1}$, se inoculó las semillas del algodón transgénico Bollgard®. El experimento consistió en los siguientes tratamientos: a) semillas sin inocular y b) semillas inoculadas con T. longibrachiatum ICA-4. Cada tratamiento consistió en bloques completamente aleatorizados. Cada unidad experimental fue de $15 \times 25 \mathrm{~m}$, con una distancia entre surcos de 1,5 m. La fertilización se hizo de acuerdo a lo sugerido por López-Martínez et al. (2014), para el cultivo de algodón Bollgard®. La emergencia de las plántulas se registró 7 días después de la siembra (DDS). De cada parcela experimental 90 DDS, se colectó de manera aleatoria 50 plántulas inoculadas y 50 no inoculadas, de cada parcela para la determinación de la enzima fenilalanina 
amonio-liasa (PAL), peroxidasa (POX) y compuestos fenólicos totales.

\section{Determinación de los compuestos fenólicos en plantas hojas}

El contenido de compuestos fenólicos totales se determinó usando el método de Folin-Ciocalteu reportado por Núñez-Ramírez et al. (2011) con modificaciones. Para ello, $50 \mathrm{~g}$ (hojas de algodón) de cada tratamiento se expusieron a metanol $80 \%$ $(1 / 10)$, se sonicó en un baño ultrasónico $\left(\mathrm{VWR}^{(\mathrm{R})}\right.$ $50 \mathrm{~Hz}$ ) durante 3 min y se centrifugó a $4.000 \mathrm{rpm}$ durante 5 min a $4{ }^{\circ} \mathrm{C}$. Posteriormente, $1 \mathrm{~mL}$ de la solución del extracto se mezcló con $5 \mathrm{~mL}$ de Folin Ciocalteu (Sigma-Aldrich, St Louis, MO) y $4 \mathrm{~mL}$ de $\mathrm{Na}_{2} \mathrm{CO}_{3}(1 \mathrm{M})$. La mezcla se agitó en vórtex y se incubó a temperatura ambiente en oscuridad durante $30 \mathrm{~min}$. Finalizado el periodo de incubación se midió la absorbancia a $765 \mathrm{~nm}$ usando un espectrofotómetro (GENESYS TM 20). La concentración total de compuestos fenólicos se calculó utilizando una curva estándar de ácido gálico. Los valores totales de fenol se expresaron como mg equivalentes de ácido gálico (GAE) por $100 \mathrm{~g}$ de peso fresco (PF).

\section{Determinación de flavonoides en plantas de hojas}

El contenido de flavonoides presentes en las hojas de algodón se determinó cuantitativamente mediante el ensayo colorimétrico de cloruro de aluminio de acuerdo a la metodología propuesta por Chang et al. (2002) usando quercetina como estándar (Sigma-Aldrich, St Louis, MO). La absorbancia de la mezcla de reacción se midió a $415 \mathrm{~nm}$ en un espectrofotómetro (GENESYS ${ }^{\mathrm{TM}}$ 20). El contenido de flavonoides totales se expresó como equivalentes de quercetina en $\mathrm{mg} \mathrm{g}^{-1}$ peso fresco (QE $\left.\mathrm{mg} \mathrm{g}^{-1} \mathrm{PF}\right)$.

\section{Determinación de la actividad peroxidasa (POX)}

Para la extracción de la enzima, 200 mg de hojas por triplicado, se homogenizaron usando un mortero previamente enfriado en $100 \mathrm{mM}$ de tampón Tris-HCl pH 7. Posteriormente, el homogenizado se colectó y centrifugó a 10.000 rpm durante $15 \min$ a $4^{\circ}$. El sobrenadante se utilizó como extracto enzimático crudo. La actividad de la peroxidasa se evalúo de acuerdo a Koksal y Gulcin (2008). Para tal fin se preparó una mezcla de reacción de $3 \mathrm{~mL}$ que consistió de una solución con $50 \mu \mathrm{L}$ del extracto de enzima, $250 \mu \mathrm{L}$ de guaiacol al $0,25 \%$ en $100 \mathrm{mM}$ Tris- $\mathrm{HCl}$ pH 7,0 tampón y $0,125 \%$ de $\mathrm{H}_{2} \mathrm{O}_{2}$. La actividad de la peroxidasa en los extractos se midió como un aumento en la absorbancia a 470 y se calculó usando el coeficiente de extinción molar de guayacol $\left(5,570 \times 10-6 \mathrm{~mol} \mathrm{~mL}^{-1}\right)$. Una unidad de actividad (UPOX) se definió como el cambio en una unidad de absorbancia por min. La actividad específica se expresó en unidades de actividad por $\mathrm{mg}$ de proteína (UPOX/mg proteína).

\section{Determinación de la actividad de la fenilalanina amonio-liasa (PAL)}

La actividad PAL se determinó mediante la evaluación de la producción de ácido cinámico-t a $290 \mathrm{~nm}$ de acuerdo a Rodríguez et al. (2006), usando $400 \mathrm{mg}$ de hojas que se homogeneizaron en $5 \mathrm{~mL}$ de $100 \mathrm{mM}$ de tampón de borato de sodio (pH 8,5). El homogeneizado se centrifugó a 12.000 rpm durante 15 min a $4{ }^{\circ} \mathrm{C}$ y el sobrenadante fue utilizado para ensayos enzimáticos. La actividad PAL se evaluó en una solución de reacción $(1 \mathrm{~mL})$ compuesta de $50 \mathrm{mM}$ de tampón de borato de sodio ( $\mathrm{pH} 8,5), 20 \mathrm{mM}$ de L-fenilalanina y el extracto de enzima. La muestra se incubó a $35^{\circ} \mathrm{C}$ y la reacción se detuvo después de $1 \mathrm{~h}$ por adición de $0,5 \mathrm{~mL}$ de ácido tricloroacético al $10 \%$. Por último, la absorbancia a A290 nm se midió después de $1 \mathrm{~h}$. Una unidad de actividad enzimática se definió como la cantidad de enzima que causa la disminución de la absorbancia de 0,01 por min. La actividad específica de PAL fue expresada en unidades de actividad por $\mathrm{mg}$ de proteína (UPAL/mg proteína).

\section{Determinación de proteínas}

La concentración de proteína se cuantificó por el método de Bradford (1976), usando albúmina sérica bovina como estándar.

\section{Análisis estadístico}

El análisis de los datos obtenidos se realizó utilizando la prueba t de Student con un nivel de 
significación del contraste: $\alpha \leq 0,05$. Se utilizó el paquete estadístico Statistica AX versión 6.1.

\section{Resultados y Discusión}

La protección química es una de las primeras respuestas estructurales de las plantas frente al ataque de patógenos o frente algún tipo de estrés, en donde los compuestos fenólicos juegan un papel importante, ya que estos desempeñan importantes funciones de protección contra patógenos (Bhattacharya et al., 2010). Se observó que el contenido de compuestos fenólicos del tejido foliar de plantas tratadas previamente con T. longibrachiatum cepa ICA-4, presentaron un incremento significativo del $18 \%$ de fenoles totales y un $45 \%$ en los niveles de flavonoides con respecto a las plantas no tratadas (Tabla 1). El incremento de los compuestos fenólicos en el tejido foliar de las plantas de algodón inoculadas con T. longibrachiatum cepa ICA-4, indica la expresión de una resistencia sistémica inducida (IRS). Similares resultados han sido previamente reportados por Shoresh et al. (2010), quienes indican la importancia del uso cepas de Trichoderma como inductores de resistencia sistémica en un amplio número de plantas de interés agrícola. En el caso de T. longibrachiatum, la información es limitada, ya que esta se ha empleado principalmente como inoculante en mejoras del proceso de fermentación de silos de alfalfa (Nadeau et al., 2000). Aunque trabajos recientes muestran la presencia de un efecto antagónico de T. longibrachiatum en condiciones in vitro sobre algunos aislados de Fusarium verticillioides (Nirenberg) procedentes de raíces de maíz (Sobowale et al., 2010). La inoculación del T. longibrachiatum en algodón generó un incremento significativo en el tejido foliar la actividad de la enzima POX de $4,88 \times 10^{-5}$ (U/mg proteína), con respecto a las plantas no inoculadas $\left(3,48 \times 10^{-5} \mathrm{U} /\right.$ mg proteína). De la misma manera se observó que la actividad de la enzima PAL en el tejido foliar de plantas de algodón previamente inoculadas con T. longibrachiatum presentaron una mayor actividad de PAL mostrando valores de 0,0176 (U/ mg proteína), que fue significativamente mayor cuando se compara con respecto a las plantas testigo (Tabla 2). El incremento de compuestos fenólicos en el tejido foliar de plantas de algodón tratadas con $T$. longibrachiatum cepa ICA-4, podría tener un efecto bioprotector contra fitopatógenos del suelo. Rodríguez-Maturino et al. (2015), sugieren que el incremento de compuestos fenólicos podría interactuar con la pared celular de los hongos fitopatógenos inhibiendo algunas enzimas fúngicas, afectando el crecimiento del micelio y disminuyendo la severidad de la enfermedad. El incremento de la enzima POX en el tejido foliar de plantas inoculadas

Tabla 1. Contenido de compuestos fenólicos y flavonoides en plantas de algodón inoculadas con T. longibrachiatum, a los 90 días después de la siembra.

\begin{tabular}{lcc}
\hline \multicolumn{1}{c}{ Tratamientos } & $\begin{array}{c}\text { Compuestos fenólicos } \\
\text { (mg GAE/100 g PF) }\end{array}$ & $\begin{array}{c}\text { Flavonoides } \\
(\mathrm{QE} \mathrm{mg/g} \mathrm{PF)}\end{array}$ \\
\hline Algodón sin inocular (Control) & $33,46 \pm 0,97^{\mathrm{a}}$ & $880 \pm 37,24^{\mathrm{a}}$ \\
Algodón inoculado & $39,45 \pm 0,70^{\mathrm{b}}$ & $1.402,89 \pm 145,71^{\mathrm{b}}$ \\
\hline
\end{tabular}

Valores con letras diferentes en la misma columna son significativamente diferentes $(\alpha \leq 0,05)$ de acuerdo a la prueba de $t$ de Student. Los resultados son medias \pm D.S. de 3 réplicas para cada tratamiento.

Tabla 2. Actividad enzimática de peroxidasa (POX) y fenilalanina amonio-liasa (PAL) en plantas de algodón inoculadas con T. longibrachiatum, a los 90 días después de la siembra.

\begin{tabular}{llc}
\hline \multicolumn{1}{c}{ Tratamientos } & $\begin{array}{c}\text { Actividad de POX } \\
\text { (UPOX/mg proteína) }\end{array}$ & $\begin{array}{r}\text { Actividad de PAL } \\
\text { (PAL/mg proteína) }\end{array}$ \\
\hline Algodón sin inocular (Control) & $3,48 \times 10^{-5} \pm 5.375,88^{\mathrm{a}}$ & $0,010 \pm 0,003^{\mathrm{a}}$ \\
Algodón inoculado & $4,88 \times 10^{5} \pm 3.874,29^{\mathrm{b}}$ & $0,017 \pm 0,004^{\mathrm{b}}$ \\
\hline
\end{tabular}

Valores con letras diferentes en la misma columna son significativamente diferentes $(\alpha \leq 0,05)$ de acuerdo a la prueba de $\mathrm{t}$ de Student. Los resultados son medias \pm D.S. de 3 réplicas para cada tratamiento. 
con T. longibrachiatum cepa ICA-4, podría favorecer la resistencia mecánica de las paredes celulares, como resultado de una mayor síntesis de lignina, limitando el avance del patógeno. Lo anterior ha sido reportado previamente en plantas de palma datilera (Phoenix dactilifera) que al ser inoculadas con T. harzianum, las que presentaron mayor tolerancia al ataque de patógenos (Khaled et al., 2008). Asimismo, T. longibrachiatum podría activar la presencia de PAL en las plantas, como resultado de la inducción de enzimas como celulasas y otras ligadas al mecanismo de estrés oxidativo que activarían diferentes rutas metabólicas, como la vía de síntesis del ácido salicílico, jasmónico y acumulación de etileno, que estimularían el metabolismo de los fenilpropanoides, reduciendo el daño por patógenos (Martinez et al., 2001; Tucci et al., 2013). Aun cuando la inducción de enzimas relacionadas con mecanismos de defensa de las plantas, como POX y PAL, ha sido previamente reportado, principalmente al emplear cepas de T. harzianum o T. viride (Singh y Singh, 2014). Los estudios de T. longibrachiatum en la activación de enzimas de defensa y antioxidantes en plantas de interés agrícola en México, son escasos. La cepa nativa de T. longibrachiatum estimuló la inducción de POX y PAL, en plantas de algodón transgénico en condiciones de campo, lo que coincide con reportes previos, Esta cepa ha mostrado efecto en el desarrollo vegetativo en plantas de algodón (Vargas-Bejarano et al., 2012), quien muestra el potencial de esta cepa de ser empleada como una herramienta biotecnológica alterna al uso de agroquímicos en el cultivo del algodón en las condiciones semiáridas del valle de Mexicali, Baja California.

\section{Conclusiones}

La inoculación de T. longibrachiatum cepa ICA-4 en algodón transgénico induce la producción de compuestos fenólicos en plantas de algodón transgénico, así como una mayor actividad específica de enzimas de relacionadas con la defensa de las plantas a patógenos, tal como PAL y POX, en condiciones de campo.

Son necesarios nuevos estudios para evaluar el impacto de $T$. longibrechiatum cepa ICA-4 en la inducción de genes de la ruta fenilpropanoide y enzimas relacionadas con la inducción de resistencia sistémica.

\section{Agradecimientos}

Se agradece a la Universidad Autónoma de Baja California, por el apoyo otorgado por medio de la $18^{\mathrm{a}}$ Convocatoria Interna de Proyectos de Investigación.

\section{Literatura Citada}

Bhattacharya, A.; Sood, P.; Citovsky, V.

2010. The roles of plant phenolics in defence and communication during Agrobacterium and Rhizobium infection. Molecular Plant Pathology, 11: 705-719.

Bradford, M.

1976. A rapid and sensitive method for the quantitation of microgram quantities of protein utilizing the principle of protein-dye binding. Analytical Biochemistry, 72: 248-254.

Chang, C.H.; Yang, M.; Wen, H.; Chern, J.

2002. Estimation of total flavonoid content in Propolis by two complementary colorimetric methods. Journal of Food and Drug Analisis, 10: 178-182.

Chowdappa, P.; Kumar, S.P.M.; Lakshmi, M.J.; Upreti, K.K. 2013. Growth stimulation and induction of systemic resistance in tomato against early and late blight by Bacillus subtilis OTPB1 or Trichoderma harzianum OTPB3. Biological Control, 65: 109-117.

Harman, G.E.; Howell, C.R.; Viterbo, A.; Chet, I.; Lorito, M. 2004. Trichoderma spp.,opportunistic avirulent plant symbionts. Nature Microbiology Review, 2: 43-56.

Karademir, A.; Aydemir, C.; Yenidoğan, S.

2011. Sound absorption and print density properties of recycled sheets made from waste paper and agricultural plant fibres. African Journal of Agricultural Research, 6: 6073-6081.

Khaled, L.B.; Pérez-Gilabert, M; Dreyer, B.; Oihabi, A.; Honrubia M.; Morte, A.

2008. Peroxidase changes in Phoenix dactylifera palms inoculated with mycorrhizal and biocontrol fungi. Agronomy for Sustainable Development, 28: 411-418.

Koksal, E.; Gulcin I.

2008. Purification and characterization of peroxidase from cauliflower (Brassica oleracea $\mathrm{L}$. var. botrytis) buds. Protein and Peptide Letters, 15: 320-326.

Li, X.G.; Liu, B.; Heia, S.; Liu, D.D.; Han, Z.M.; Zhou, K.X.; Cui, J.J.; Luo, J.Y.; Zheng Y.P.

2009. The effect of root exudates from two transgenic insect-resistant cotton lines on the growth of Fusarium oxysporum. Transgenic Research, 18: 757-767.

Li, X.G.; Wei, Q.; Liu, B.; Alam, M.S.; Wang, X.X.; Shen, W.; Han, Z.M.

2013. Root exudates of transgenic cotton and their effects on Fusarium oxysporum. Frontier in Bioscience, 1: 725-33. López Martínez, J.D.; Salazar Sosa, E.; Trejo-Escareño, H.I.; García Hernández, J.L.; Navarro Morones, M.; Vázquez-Vázquez, C. 2014. Producción de algodón con altas densidades de siembra usando fertilización orgánica. Phyton, 83: 237-242. 
Martínez, C.; Blanc, F.; Le Claire, E.; Besnard, O.; Nicole, M.; Baccou, J.C.

2001. Salicylic acid and ethylene pathways are differentially activated in melon cotyledons by active or heat-denatured cellulase from Trichoderma longibrachiatum. Plant Physiology, 127: 334-344.

Nadeau, E.M.; Russell, J.R.; Buxton, D.R.

2000. Intake, digestibility, and composition of orchardgrass and alfalfa silages treated with cellulase, inoculant, and formic acid fed to lambs. Journal Animal Science, 78: 2980-2989.

Nawrocka, J.; Malolepsza, U.

2013. Diversity in plant systemic resistance induced by Trichoderma. Biological Control, 67: 149-156.

Núñez-Ramírez, F.; González-Mendoza, D.; Grimaldo-Juárez, O.; Díaz, L.C.

2011. Nitrogen fertilization effect on antioxidants compounds in fruits of habanero chili pepper (Capsicum chinense). International Journal of Agriculture and Biology, 13: 827-830.

Rajendran, L.; Samiyappan, R.

2008. Endophytic Bacillus species confer increased resistance in cotton against damping off disease caused by Rhizoctonia solani. Journal of Plant Pathology, 7: 1-12.

Rodríguez, Y.; Fernández, A.; Solórzano, E.; Peteira, B.; Fernández, F. 2006. Inducción de enzimas de defensa en dos variedades de arroz (Oryza sativa) por el hongo micorrízico arbuscular $G$. mosseae. Revista Colombiana de Biotecnología, 3: 35-49.

Rodríguez-Maturino, A.; Troncoso-Rojas, R.; Sánchez-Estrada, A.; González-Mendoza, D.; Ruiz-Sánchez, E.; Zamora-Bustillos,

R.; Cecena-Durán, C.; Grimaldo-Juárez, O.; Aviles-Marín, M. 2015. Efecto antifúngico de extractos fenólicos y de carotenoides de chiltepín (Capsicum annum var. glabriusculum) en Alternaria alternata y Fusarium oxysporum. Revista Argentina de Microbiología, 47: 72-77.
Shoresh, M.; Harman, G.; Mastouri, F.

2010. Induced Systemic Resistance and Plant Responses to Fungal Biocontrol Agents. Annual Review Phytopathology, 48: 21-43.

Singh, S.P.; Singh, H.B.

2014. Effect of mixture of Trichoderma isolates on biochemical parameters in leaf of Macrophomina phaseolina infected brinjal. Journal Environmental Biology, 35: 871-876.

Sobowale, A.A.; Odebode, A.C.; Cardwell, K.F.; Banyopadhyay, R.; Jonathan, S.G.

2010. Antagonistic potential of Trichoderma longibrachiatum and $T$. hamatum resident on maize (zea mays) plants against Fusarium verticillioides (Nirenberg) isolated from rotting maize stem. Archives of Phytopathology and Plant Protection, 8: 43: 744-753.

Terán-Vargas, A.P.; Rodríguez, J.C.; Blanco, C.A.; MartínezCarrillo, J.L.; Cibrián-Tovar, J.; Sánchez-Arroyo, H.; Rodríguez-Del-Bosque, L.A.; Stanley, D.

2005. Bollgard cotton and resistance of tobacco budworm (Lepidoptera: Noctuidae) to conventional insecticides in the Southern Tamaulipas, Mexico. Journal of Economic Entomology, 98: 2203-2209.

Tucci, M.; Ruocco, M.; De Masi, L.; De Palma, M.; Lorito, M. 2011. The beneficial effect of Trichoderma spp. on tomato is modulated by the plant genotype. Molecular Plant Pathology, 12: 341-354.

Vargas-Bejarano, E.; Méndez-Trujillo, V.; Vázquez Angulo, J.C.; González-Mendoza, D.; Grimaldo Juárez, O.

2012. Cambios fisiológicos en algodón transgénico inoculado con Trichoderma spp. Phyton, 81: 101-105.

Zahoor, O.; Ahmad, S.; Fazli Raziq, H.; Khan, Idrees M.

2012. Chemical and Biological Control of fusarium root rot of okra. Pakistan Journal of Botany, 44: 453-457. 